\title{
Keyword Index Volume 23 (2011)
}

\author{
accessibility \\ accessibility \\ accessible ICT \\ Alzheimer's disease \\ ambient assisted living \\ appearance \\ Arabic Sign Language \\ assistive device \\ assistive technology
}

background info

blind

challenges

clothing matching

color blind

color matching

computer vision

cursor-positioning

database

design for all

disability

disability products

dynamic pointing assistive program

eAccessibility

eInclusion

e-inclusion

emancipatory research

entrepreneurship

Europe

European policies

framework

geo-positioning technologies

global positioning system

gravity balance
139

223

171

243

7

191

65

191

$1,7,41,53,87,101$,

$131,149,163,171,183$

149

75

41

75

75

75

75

215

233

$101,149,163$

7, 41, 139, 191, 215, 223, 243

53

215

223

101

183

87

53

7,131

183

1

7

19 hearing impaired $\quad 65$

HEART 183

higher education $\quad 163$

ICF 1

ICT 41

inclusion 41

inclusive design 163

independent living $\quad 7$

information 205

instrument 1

$\begin{array}{ll}\text { internet } & 7\end{array}$

legislation $\quad 139$

$\begin{array}{ll}\text { location-aware services } & 7\end{array}$

mild cognitive impairment 243

mobile phones 65

mouse driver 215

mouse wheel 215

MPT (Matching Person and Technology) 191

multi-modality 233

natural language processing $\quad 65$

occupational therapy 243

older adults 243

$\begin{array}{ll}\text { older people } & 7\end{array}$

pattern analysis $\quad 75$

phenomenography 205

policy 139

practitioner 1

$\begin{array}{ll}\text { procurement } & 149\end{array}$

$\begin{array}{ll}\text { product design } & 163\end{array}$

qualitative analysis 205

quality 131

regulation 191

research and development $\quad 183$ 
road-mapping

selection

self-image

service delivery

sign language

signing avatar

social entrepreneurship

social model of disability

standardisation

structured dialogue

technical aid
Keyword Index Volume 23 (2011)

171 technology transfer

telehealthcare

191 universal design 163

1, 131 user involvement 149

233

65 visually impaired 75

83 web accessibility 223

149 web design 223

171 web development 223

Wikipedia

233

191 